\title{
Evaluación del consumo en antidepresivos en la población uruguaya entre 2010 y 2014
}

Evaluation of antidepressant use in the Uruguayan population between 2010 and 2014 Avaliação do uso de antidepressivos na população uruguaia entre 2010 e 2014

\author{
Emiliano Pagano¹, Viviana Domínguez², Noelia Speranza³ \\ Leandro Barboza ${ }^{4}$, Gustavo Tamosiunas ${ }^{5}$
}

\section{Resumen}

Objetivo: conocer el consumo de antidepresivos en la población uruguaya en el período 2010-2014.

Material y método: se realizó un estudio de utilización de medicamentos para evaluar el consumo de antidepresivos utilizando el dato de dispensación de medicamentos de las farmacias de las instituciones participantes. Se utilizó la variable dosis diaria definida (DDD) por 1.000 habitantes/día (DHD).

Resultados: se incluyó el 69\% de la población uruguaya. Las DHD globales de antidepresivos para los años 2010, 2011, 2012, 2013 y 2014 fueron: 26,49, 29,71, 30,17, 30,79 y 32,55 (promedio en los 5 años: 30,04) siendo el aumento porcentual global en dicho período de 22,88\%. Los ISRS fueron el grupo de antidepresivos más consumidos. Sertralina fue el antidepresivo más consumido en los 5 años de estudio (DHD 13,65), y el de mayor aumento porcentual fue duloxetina, seguido por escitalopram.

Conclusión: el consumo de antidepresivos a nivel nacional en el período analizado es inferior al constatado en otros países, con una tendencia al aumento. Éste puede ser visto como una señal para la evaluación evolutiva en el periodo 2015-2020 y para realizar análisis de las prácticas de prescripción y actuales indicaciones, utilizando otras metodologías como los estudios de prevalencia o estudios de indicación-prescripción o de prescripción-indicación.

Palabras clave: Antidepresivos

Key words: $\quad$ Antidepressive agents

\footnotetext{
1. Ex Asist. Departamento de Farmacología y Terapéutica, Facultad de Medicina, Universidad de la República (UdelaR);

2. Ex Prof. Adj. Departamento de Farmacología y Terapéutica, Facultad de Medicina, Universidad de la República (UdelaR);

3. Prof. Agda. Departamento de Farmacología y Terapéutica, Facultad de Medicina, Universidad de la República (UdelaR);

4. Asist. Departamento de Farmacología y Terapéutica, Facultad de Medicina, Universidad de la República (UdelaR);

5. Prof. Departamento de Farmacología y Terapéutica, Facultad de Medicina, Universidad de la República (UdelaR);

Departamento de Farmacología y Terapéutica, Facultad de Medicina, Universidad de la República (UdelaR). Hospital de Clínicas.

Los autores declaran no tener conflicto de intereses.

Correspondencia: Dr. Emiliano Pagano. Correo electrónico: emiliano464@gmail.com

Recibido: 11/12/2020

Aprobado: 8/3/2021
} 


\section{Introducción}

Los antidepresivos son el tratamiento de primera línea para la depresión unipolar de intensidad moderada a grave (para las depresiones leves estarían indicados solo en algunas situaciones) y para los trastornos de ansiedad según varias guías clínicas ${ }^{(1,2)}$. La depresión y los trastornos de ansiedad se caracterizan por tener una elevada frecuencia a nivel mundial ${ }^{(3)}$. Según la Organización Mundial de la Salud (OMS) la depresión es la principal causa de pérdida de salud dentro de las enfermedades no fatales mientras que los trastornos de ansiedad se ubican en el sexto lugar ${ }^{(3)}$. En Uruguay la prevalencia para 2015 estimada según la Organización Mundial de la Salud (OMS) para depresión fue de 5\% y de $6,4 \%$ para los trastornos de ansiedad, cifras algo mayores que las estimadas a nivel mundial $(4,4 \%$ y $3,6 \%$ respectivamente $)^{(4)}$. Ambos trastornos tienen consecuencias graves tanto para las personas que los padecen como para la sociedad en su conjunto. Además, tanto la depresión como los trastornos de ansiedad son un factor de riesgo para el suicidio, fenómeno complejo y evitable, que constituye en Uruguay un problema de salud pública por su elevada frecuencia y en aumento creciente (tasa cada 100.000 habitantes de 2010 a 2014 fue de 16,19, 17,74, 16,13 y 17,40 respectivamente, siendo para el año 2019 de 20,55 cada 100.000 habitantes $)^{(5)}$.

Varios factores pueden incidir en la forma en que se prescriben los medicamentos, que no siempre se produce de forma racional y razonada, por lo que la OMS recomienda realizar una monitorización continua y activa de su utilización mediante estudios de uso de medicamentos (EUM) $)^{(6,7)}$. Este tipo de estudios son, junto a la farmacovigilancia, fuentes de información relevante para ayudar a comprender las consecuencias médicas, sociales y económicas del uso de medicamentos. Complementariamente a estos tipos de análisis es importante contar también con datos cualitativos sobre el modo de uso. Los estudios de consumo a través del cálculo de indicadores como la dosis diaria definida (DDD) por mil habitantes día (DHD) o cada 100 camas día son ejemplo de ellos. La DDD es una unidad técnica de medida fija que corresponde a la dosis promedio de mantenimiento en la principal indicación para una vía de administración determinada en adultos, independiente del precio o del envase. La DHD es un indicador de mayor utilidad para medicamentos que se prescriben en forma crónica y sirve principalmente como medida de comparación entre dos poblaciones o en la misma población en dos momentos distintos. No refleja necesariamente la dosis diaria realmente prescrita o utilizada por cada paciente y puede utilizarse como estimativo de la prevalencia de la enfermedad si el fármaco en cuestión es prescrito para una sola indicación ${ }^{(8)}$.

Faltan estudios que caractericen la utilización de los antidepresivos a nivel nacional. La séptima Encuesta Nacional en Hogares sobre Consumo de Drogas constató que $14 \%$ de la población encuestada consumió en alguna oportunidad algún antidepresivo en su vida, y un $6,8 \%$ lo hizo en los últimos 12 meses $^{(9)}$. Por otro lado un EUM realizado en un hospital de tercer nivel en Montevideo en el período 2010-2014 con pacientes ambulatorios detectó un aumento en el consumo de antidepresivos de $8 \%$ (de 41,6 DHD/1.000/día en 2010 a 45 DHD/1.000/día en 2014) ${ }^{(10)}$.

El Ministerio de Salud Pública (MSP) evaluó el uso de medicamentos controlados durante el periodo enero-octubre 2018 mediante el reporte mensual declarado de los establecimientos autorizados para manejar este grupo de medicamentos, utilizando la DHD como indicador de consumo. Teniendo en cuenta que los resultados de este estudio fueron parciales, no solo porque no completan un año sino porque los autores destacan una importante subdeclaración de la dispensación, Uruguay presentaría un consumo de psicofármacos y estupefacientes menor que el de otros países, con una DHD de 39 para benzodiazepinas, 21 para antidepresivos, y 14 para antipsicóticos ${ }^{(11)}$.

A nivel internacional, la evolución 2000-2013 en España mostró un aumento de 200\% (26,5 DHD/1.000 habitantes día en 2000 a 79,5 en 2013), mientras que Portugal aumentó su consumo de 18,7 en 1995 a 74,7 en 2009 y en Inglaterra se constató un aumento de $165 \%$ en la prescripción de antidepresivos entre 1998 y 2012. En Estados Unidos 12\% de la población mayor de 12 años consume antidepresivos, y a nivel regional Chile aumentó el consumo de este grupo de medicamentos $470 \%$ desde 1992 a $2004^{(12-16)}$.

Desde el Observatorio de Medicamentos del Departamento de Farmacología y Terapéutica, que pretende conocer la situación de prescripción y consumo de medicamentos, específicamente de psicofármacos en nuestro país, y contribuir a su uso más racional, realizando estudios multidimensionales que incluyen distintos aspectos vinculados al uso de medicamentos en Uruguay $^{(17)}$.

El objetivo del presente estudio fue conocer el consumo de antidepresivos en la población uruguaya en el período 2010-2014.

\section{Metodología}

Se realizó un EUM para evaluar el consumo de antidepresivos a través del dato de dispensación de las farmacias de las instituciones de salud participantes. 
El consumo se determinó utilizando la variable dosis diaria definida (DDD) por mil habitantes/día (DHD). La DHD se puede interpretar como el número de habitantes por cada 1000 que recibió una DDD cada día de un año determinado. La fórmula utilizada fue ${ }^{(18)}$.

$\mathrm{DHD}=\quad \mathrm{n}^{\mathrm{o}} \mathrm{DDD} \times 1.000$ habitantes $\mathrm{n}^{\circ}$ habitantes en la población $\mathrm{x}$ tiempo (días)

donde:

$\mathrm{n}^{\circ} \mathrm{DDD}=\mathrm{mg} / \mathrm{DDD}(\mathrm{mg})$

$\mathrm{mg}=$ suma de miligramos ( $\mathrm{mg}$ ) de antidepresivos dispensados en 1 año

$\mathrm{DDD}=$ dosis diaria definida según OMS cita tiempo $=365$ días

$\mathrm{n}^{\circ}$ habitantes $=$ número total de usuarios de las instituciones de salud

Los antidepresivos analizados fueron todos los comercializados a nivel nacional al 2015: moclobemida, amitriptilina, clomipramina, imipramina, nortriptilina, citalopram, maprotilina, citalopram, escitalopram, fluoxetina, fluvoxamina, paroxetina, sertralina, duloxetina, venlafaxina, desvenlafaxina, mirtrazapina, bupropion, agomelatina y trazodona.

Se subclasificaron según el grupo ATC (Anatomical Therapeutic Chemical Classification) en: inhibidores monoaminérgicos no selectivos ("antidepresivos tricíclicos"); inhibidores selectivos de la recaptación de serotonina (ISRS); inhibidores de la monoaminooxidasa no selectivos (IMAO); Inhibidores selectivos de la monoaminoxidasa A y otros antidepresivos. Los antidepresivos duales y otros antidepresivos no duales fueron clasificados por criterios farmacodinámicos en "otros antidepresivos",(19).

Los miligramos de todos los antidepresivos se obtuvieron de datos de dispensación de los servicios de farmacias de las instituciones de salud públicas y privadas participantes.

Se solicitó información a 13 instituciones de salud del país. La selección de las mismas se realizó en base a la población de usuarios que atienden y a la accesibilidad a los datos requeridos. El número de habitantes se calculó en base al total de usuarios de las instituciones incluidas, por año. Los datos provistos por cada institución fueron registrados en una base de datos prediseñada en Excel.

Se calculó la DHD para cada antidepresivo para cada año del período incluido. Para conocer el DHD global anual se realizó la sumatoria de las DHD de todos los antidepresivos de todas las instituciones participantes. Los datos se expresaron en valores absolutos y como cam- bios porcentuales. Se calculó una DHD global para todos los antidepresivos y se evaluó su variación en el periodo de estudio considerado. Se realizó un subanálisis según grupos terapéuticos de antidepresivos ${ }^{(19)}$.

\section{Resultados}

Se analizaron los datos de dispensación de las 13 instituciones de salud incluidas, una de ellas representa al principal prestador de salud pública del país (Administración de los Servicios de Salud Del Estado, ASSE) y el resto al subsector privado. Cuatro de ellas pertenecían al interior del país. El total de usuarios de las instituciones participantes representan promedialmente $68,97 \%$ de la población uruguaya en los 5 años inclui$\operatorname{dos}^{(20)}$

De los 19 antidepresivos incluidos, 11 se encontraban disponibles en todas las instituciones, todos los años analizados.

Las DHD globales de antidepresivos para los años 2010, 2011, 2012, 2013 y 2014 fueron: 26,49, 29,71, $30,17,30,79$ y 32,55 (promedio en los 5 años: 30,04 ) siendo el aumento porcentual global en dicho período de $22,88 \%$. El consumo por grupo terapéutico y antidepresivo por año se describe en la tabla 1 .

Todos los antidepresivos, a excepción de los antidepresivos tricíclicos, fluoxetina Y moclobemida, aumentaron su consumo. Los ISRS fueron el grupo de antidepresivos más consumidos. El aumento porcentual más alto del período fue para el grupo "otros antidepresivos", que incluye los duales y otros no duales (tabla 1).

Sertralina fue el antidepresivo más consumido en los 5 años de estudio, y el de mayor aumento porcentual fue duloxetina, seguido por escitalopram.

\section{Discusión}

Este es un trabajo de base poblacional para evaluar el consumo de antidepresivos en Uruguay. La población incluida en el análisis corresponde a más de $60 \%$ de la población uruguaya. Si bien no se realizó un muestreo, sino una selección por conveniencia, representa más de la mitad de la población del país. La DHD para el consumo de todos los antidepresivos entre 2010-2014 fue de 30,04 , con una tendencia al aumento en dicho período de casi el $23 \%$.

Es probable que el valor de consumo hallado esté subestimado dado que solo considerando la prevalencia de la depresión y el trastorno de ansiedad generalizada referidas previamente éste debería ser mayor ${ }^{(4)}$. Aún más, la frecuencia podría ser aún mayor si se tomaran en cuenta otros datos disponibles a nivel local ${ }^{(21)}$.

También es posible que el consumo de antidepresivos sea bajo a nivel nacional, en concordancia con lo que indica la OMS que en la región de las Américas 7 de ca- 
Tabla 1. Consumo de antidepresivos, por grupo terapéutico y fármaco, por año y cambio porcentual, 2010-2014.

\begin{tabular}{|c|c|c|c|c|c|c|}
\hline Antidepresivos (ADP) & DHD 2010 & DHD 2011 & DHD 2012 & DHD 2013 & DHD 2014 & $\begin{array}{c}\text { Cambio } \\
\text { porcentual } \\
2010-2014\end{array}$ \\
\hline \multicolumn{7}{|c|}{ Inhibidores no selectivos de la recaptación de monoaminas (ADT; ATC NO6AA) (ADP tricíclicos) } \\
\hline Amitriptilina & 0,67 & 0,73 & 0,7 & 0,73 & 0,59 & $-11,94$ \\
\hline Clomipramina & 0,14 & 0,14 & 0,13 & 0,12 & 0,11 & $-21,43$ \\
\hline Imipramina & 0,22 & 0,23 & 0,19 & 0,15 & 0,15 & $-31,82$ \\
\hline Maprotilina & 0,03 & 0,03 & 0,02 & 0,02 & 0,02 & $-33,33$ \\
\hline Nortriptilina & 0,00 & 0,00 & 0,00 & 0,00 & 0,00 & 0,00 \\
\hline Sub-total ADT & 1,06 & 1,13 & 1,04 & 1,02 & 0,87 & $-17,92$ \\
\hline \multicolumn{7}{|c|}{ Inhibidores selectivos recaptación de serotonina (ISRS; ATC NO6AB) } \\
\hline Fluoxetina & 5,91 & 5,72 & 5,3 & 4,78 & 4,78 & $-19,12$ \\
\hline Sertralina & 11,58 & 13,25 & 14,03 & 14,32 & 15,11 & 30,48 \\
\hline Escitalopram & 1,47 & 1,86 & 2,32 & 2,38 & 3,08 & 109,52 \\
\hline Citalopram & 2,05 & 2,37 & 2,42 & 2,13 & 2,14 & 4,21 \\
\hline Paroxetina & 1,06 & 1,28 & 1,33 & 1,37 & 1,58 & 49,06 \\
\hline Fluvoxamina & 0,66 & 0,78 & 0,82 & 0,85 & 0,85 & 28,79 \\
\hline Subtotal ISRS & 22,73 & 25,26 & 26,22 & 25,83 & 27,54 & 21,16 \\
\hline \multicolumn{7}{|c|}{ Inhibidores de la monoaminoxidasa (IMAO; ATC N06AG) } \\
\hline Moclobemida & 0,01 & 0,01 & 0,00 & 0,00 & 0,00 & $-100,00$ \\
\hline \multicolumn{7}{|c|}{ Otros ADP (ATC N06AX) } \\
\hline \multicolumn{7}{|c|}{ ADP "duales" (ISRSN; ATC N06AX) } \\
\hline Desvenlafaxina & 0,00 & 0,00 & 0,00 & 0,00 & 0,00 & 0,00 \\
\hline Duloxetina & 0,06 & 0,15 & 0,22 & 0,41 & 0,57 & 850,00 \\
\hline Venlafaxina & 2,34 & 2,72 & 2,69 & 2,97 & 3,09 & 32,05 \\
\hline Subtotal ISRSN & 2,40 & 2,87 & 2,91 & 3,38 & 3,66 & 52,50 \\
\hline \multicolumn{7}{|c|}{ Otros ADP no duales (ATC N06AX) } \\
\hline Agomelatina & 0,00 & 0,06 & 0,05 & 0,08 & 0,02 & 100,00 \\
\hline Bupropión & 0,18 & 0,21 & 0,23 & 0,26 & 0,30 & 66,67 \\
\hline Mirtazapina & 0,06 & 0,10 & 0,09 & 0,04 & 0,10 & 66,67 \\
\hline Trazodona & 0,05 & 0,07 & 0,25 & 0,06 & 0,06 & 20,00 \\
\hline Subtotal otros ADP no duales & 0,29 & 0,44 & 0,62 & 0,44 & 0,48 & 65,52 \\
\hline Subtotal otros ADP (duales y no duales) & 2,69 & 3,31 & 3,53 & 3,82 & 4,14 & 53,90 \\
\hline Total ADP & 26,49 & 29,71 & 30,79 & 30,67 & 32,55 & 22,88 \\
\hline
\end{tabular}


da 10 habitantes con depresión no acceden a los tratamientos que requieren ${ }^{(22)}$. De todas formas resulta complejo, solo con los datos cuantitativos de este estudio, evaluar la pertinencia del consumo. El aporte del presente estudio es brindar un dato de pretendida base nacional para evaluar la evolución del consumo de los subgrupos de antidepresivos y para poder comparar el consumo con otro período de tiempo a nivel nacional e internacional, y así contribuir a generar la línea de base necesaria para evaluar recomendaciones, cambios u otras acciones de prescripción racional.

$\mathrm{Al}$ intentar comparar estos datos con otros datos nacionales, por ejemplo los presentados por el MSP sobre consumo de sertralina para el período enero 2014 a junio 2016 según reportes de importaciones o compra en plaza y posterior ventas a farmacias, se evidencia que es uno de los psicofármacos más consumidos luego de alprazolam, pero dada la metodología utilizada para este análisis no pueden realizarse comparaciones ${ }^{(23)}$. Otro estudio más reciente del MSP da cuenta de una DHD de antidepresivos para el año 2018 de 21, valor algo inferior al hallado en esta serie, pero nuevamente, quizás la metodología de este análisis no los haga completamente comparables, dado que los datos del MSP surgen de los balances trimestrales presentados a la autoridad sanitaria por establecimientos autorizados para manejar medicamentos controlados (farmacias privadas, farmacias hospitalarias y droguerías), habiendo recabado el dato de $49 \%$ de las que teóricamente deberían haberlo hecho ${ }^{(11)}$. Otro dato nacional, de base institucional, da cuenta de un consumo en pacientes ambulatorios antidepresivos entre 2010 y 2014 de 41,6 y 45 DHD, respectivamente.

Si se lo compara con el contexto internacional, Uruguay presentó un consumo menor de antidepresivos que España (DHD 79,5 en 2013) o Escocia (DHD 123 en 2012) $)^{(12,24)}$. Según un informe para el año 2012 de países europeos, Uruguay se encontraría para el mismo año entre los más bajos de esa región (Uruguay DHD 30,79, por debajo solo Estonia DHD 21, Hungría DHD 27 y Eslovaquia DHD 30), y superado por el resto de los países incluidos (el mayor valor es para Islandia con una DHD/1.000 habitantes día de 109) ${ }^{(25)}$. Entender los motivos de este comportamiento solo contrarrestando con los datos de prevalencia de depresión, ansiedad y suicidios en el país, merece una evaluación diferente y un análisis más profundo.

Si bien el periodo de estudio fue breve, la tendencia del consumo de antidepresivos fue al aumento al igual que en el contexto internacional, similar a lo hallado en Australia entre 2007 y $2015^{(26)}$, pero comparativamente menor que lo hallado globalmente en Europa de un aumento de 107\% (27 a 56 DHD 1.000 habitantes/día) ${ }^{(25)}$.
Dado que los antidepresivos tienen más de una indicación de prescripción sería muy complejo realizar estimaciones de prevalencia ${ }^{(8)}$. En función del valor de DHD global hallado y el análisis que hacen algunos autores de esta variable, se podría estimar que la prevalencia de la depresión es de aproximadamente 3\%. Sin embargo este valor se ubicaría algo por debajo de las estimaciones para el país, por lo que, sumado a que las dispensaciones incluidas en la cálculo no se diferencian por indicaciones (podrían haberse dispensado antidepresivos para el trastorno de ansiedad generalizada o dolor crónico) este valor podría estar subestimando la prevalencia.

En el análisis por subgrupo de antidepresivos, a pesar de la disminución en el consumo de fluoxetina, el grupo de los ISRS tuvo un aumento al igual que todos los otros grupos excepto el de los antidepresivos tricíclicos (ADT) y los inhibidores de la monoamino oxidasa (IMAO). Una de las hipótesis por las cuales se puede haber dado este fenómeno es por el "atribuido" mejor perfil de seguridad de los ISRS sobre éstos ${ }^{(27)}$. Sin embargo este hecho puede estar influenciado por el aumento de consumo en sí mismo, más que un mejor perfil de seguridad. Esta evolución del consumo es la misma a la hallada en otros países y en estudios nacionales ${ }^{(12)}$. También en concordancia con el contexto internacional, escitalopram y citalopram has sido de los antidepresivos con mayor aumento de consumo (España, Escocia) ${ }^{(12,22)}$.

La disminución de fluoxetina y el aumento de escitalopram no tiene claras explicaciones a nivel farmacoterapéutico. La aparición del escitalopram no implica gran avance terapéutico. Una revisión de la Colaboración Cochrane si bien se encontró diferencias estadísticamente significativas para la eficacia (escitalopram vs. citalopram y fluoxetina) y para la aceptabilidad (escitalopram vs. duloxetina) en el tratamiento agudo de la depresión mayor, no existía suficiente evidencia para detectar una diferencia entre escitalopram y otros antidepresivos luego de dos semanas de tratamiento y el sesgo por patrocinio puede haber incidido en la sobre estimación de los resultados positivos ${ }^{(28,29)}$.

Desvenlafaxina, duloxetina, bupropión, mirtazapina y trazodona mostraron cambios porcentuales importantes pero con números absolutos de consumo muy bajos. En cambio agomelatina presentó un máximo consumo en 2013 que luego disminuyó en 2014. Estos datos también concuerdan con los hallados en otros países como España ${ }^{(30)}$. Existieron problemas vinculados a su seguridad que pudieron haber influido en estos hallazgos ${ }^{(31,32)}$. El aumento de los ISRSN podría deberse a la aparición de duloxetina y la extensión de sus indicaciones (dolor neuropático periférico diabético y tratamiento del trastorno de ansiedad generalizada) o a la creencia de que 
los antidepresivos con mecanismo de acción "duales" son más eficaces que los ISRS. Sin embargo aún la evidencia no ha podido hallar diferencias importantes ni existen recomendaciones contundentes al respecto ${ }^{(1,33-35)}$.

El presente estudio tiene limitaciones. En primer lugar proporciona una estimación aproximada del consumo y no una fotografía exacta de la prescripción. Además se destaca que si bien fue un porcentaje importante (60\%), no se alcanzó el $100 \%$ de la población y los datos recabados reflejan valores de hace más de 5 años. Se asume además que los datos de dispensación obtenidos representan los de consumo, y esto no es estrictamente cierto. El dato de consumo a partir de dispensaciones deja por fuera del análisis los motivos por los cuales se han prescrito los antidepresivos y bajo qué pautas posológicas. Sería necesario contar con estudios de indicación prescripción para poder caracterizar mejor en qué tipo de pacientes se utilizaron. Como otra limitante tampoco se tuvieron en cuenta otros canales de dispensación de los antidepresivos como pueden ser las farmacias comunitarias o el hecho de haber recibido antidepresivos sin prescripción médica, si bien según lo que se analiza en la VII encuesta de la Junta Nacional de Drogas, $96 \%$ de las personas que consumieron antidepresivos lo hizo bajo prescripción médica ${ }^{(9)}$. También podrían analizarse, con aportes de otro tipo de estudios, si las dosis a las que se utilizan y la duración de los tratamientos son adecuados y hacer un subanálisis exclusivo de la población adulta, pues el estudio de Teixeria y colaboradores halló en su análisis de datos de una institución que el consumo en pediatría en el periodo 2010 a 2014 había disminuido $66 \%{ }^{(10)}$.

\section{Conclusiones}

Este estudio evaluó el consumo de antidepresivos a nivel nacional, siendo inferior al constatado en otros países, con una tendencia al aumento. Éste puede ser visto como una señal para la evaluación evolutiva en el período 2015-2020 y para realizar análisis de las prácticas de prescripción y actuales indicaciones, utilizando otras metodologías como los estudios de prevalencia o estudios de indicación-prescripción o de prescripción-indicación.

Es necesario mantener una monitorización continua del uso de psicofármacos para no solo conocer el comportamiento del consumo sino para hacer recomendaciones de prescripción acordes y racionales.

\section{Abstract}

Objective: to learn about antidepressant use in the Uruguayan population between 2010 and 2014 .
Method: the use of drugs was studied to evaluate the consumption of antidepressants by analysing the dispensing of drugs in the pharmacies that are part of the participating institutions. The study used the defined daily dose variable (DDD) by 1.000 inhabitants/day (DHD).

Results: $69 \%$ of the Uruguayan population was included in the study. The global antidepressant dose by inhabitants per day for 2010, 2011, 2012, 2013 and 2014 was $26,49,29,71,30,17 ; 30,79$ and 32,55 respectively (average in the five years 30,4 ) being the global increase of percentage $22,88 \% 5$ for that period.

The SSRIs (selective serotonin reuptake inhibitor) were the most widely used group of antidepressants. Sertraline was the antidepressant of greatest consumption in the 5 years of the study (DHD 13,65), and the one that presented the highest percentage increase was duloxetine, followed by escitalopram.

Conclusion: antidepressant use at the national level during the period analysed is lower than that seen in other countries, although a tendency to increase was found. This may be interpreted as a sign for the need to evaluate the evolution in the 2015-2020 period, and to conduct studies on prescription practices and current indications using other methodologies, such as prevalence studies or indication-prescription or prescription-indication studies.

\section{Resumo}

Objetivo: conhecer o consumo de antidepressivos na população uruguaia no período 2010-2014.

Material e método: foi realizado um estudo sobre o uso de medicamentos para avaliar o consumo de antidepressivos a partir dos dados de dispensação de medicamentos nas farmácias das instituições participantes. Foi utilizada a variável dose diária definida (DDD) por 1.000 habitantes/dia (DHD).

Resultados: $69 \%$ da população uruguaia foram incluídas. Os DHDs globais de antidepressivos para os anos de 2010, 2011, 2012, 2013 e 2014 foram: 26,49, $29,71,30,17 ; 30,79$ e 32,55 (média nos 5 anos, 30,04) sendo o aumento percentual global no referido período de $22,88 \%$.

Os inibidores seletivos da recaptação da serotonina foram o grupo de antidepressivos mais amplamente utilizado. A sertralina foi o antidepressivo mais utilizado nos 5 anos do estudo (DHD 13,65), e o que apresentou maior aumento percentual foi a duloxetina, seguida do escitalopram.

Conclusão: o consumo de antidepressivos em nível nacional no período analisado é inferior ao encontrado em outros países, com tendência de aumento. Isto pode ser visto como um sinal para a avaliação evolutiva no pe- 
ríodo 2015-2020 e para a realização de análises das práticas de prescrição e indicações atuais, utilizando outras metodologias como estudos de prevalência ou estudos de indicação-prescrição ou prescrição-indicação.

\section{Bibliografía}

1. National Institute for Health and Care Excellence. Antidepressant treatment in adults. NICE, 2021. Disponible en: https://pathways.nice.org.uk/pathways/depression/antidepressant-treatment-in-adults.pdf [Consulta: 26 oct 2020].

2. Katzman M, Bleau P, Blier P, Chokka P, Kjernisted K, Van Ameringen M, et al. Canadian clinical practice guidelines for the management of anxiety, posttraumatic stress and obsessive-compulsive disorders. BMC Psychiatry 2014; 14(Suppl 1):S1.

3. Organización Mundial de la Salud. Depresión. OMS, 2020. Disponible en: http://www.who.int/mediacentre/factsheets/fs369/es/ [Consulta: 26 oct 2020].

4. World Health Organization. Depression and other mental common disorders: global health estimates. WHO, 2017. Disponible en: https://apps.who.int/iris/bitstream/handle/10665/254610/WHO-MSD-MER-2017,2-eng.pdf?sequence $=1 \&$ isAllowed $=y$ [Consulta: 26 oct 2020].

5. Uruguay. Ministerio de Salud Pública. Día Nacional de Prevención del Suicidio. 17 de julio de 2020. Disponible en: https://www.gub.uy/ministerio-salud-publica/sites/ministerio-salud-publica/files/documentos/noticias/Presentaci $\%$ C3 $\%$ B3n $\% 20$ de $\% 20$ datos $\% 20$ para $\% 20 \mathrm{D} \% \mathrm{C} 3 \% \mathrm{ADa} \% 20 \mathrm{Nac} \% 20 \mathrm{P} \% 20$ Suicidio\%202020.pdf [Consulta: 26 oct 2020].

6. Tognoni G, Laporte JR. From clinical trials to drug utilization studies. En: Dukes MN, ed. Drug utilization studies: methods and uses. 1993:23-42. (WHO regional publications. European series; 45). Disponible en: https://apps.who.int/iris/handle/10665/260517 [Consulta: 26 oct 2020].

7. Haaijer-Ruskamp F, Hemminki E. The social aspects of drug use. En: Dukes MN, ed. Drug utilization studies: methods and uses. 1993:97-124. (WHO regional publications. European series; 45). Disponible en: https://apps.who.int/iris/handle/10665/260517:97-124 [Consulta: 26 oct 2020$]$.

8. Capellá D, Laporte JR. Métodos aplicados en estudios descriptivos de utilización de medicamentos. En: Laporte JR, Tognoni G. Principios de epidemiología del medicamento. 2 ed. Barcelona: Masson-Salvat, 1998:67-92.

9. Junta Nacional de Drogas. VII Encuesta nacional sobre consumo de drogas en población general. Informe de investigación. Año 2019. Disponible en: https://www.gub.uy/junta-nacional-drogas/sites/junta-nacional-drogas/files/documentos/publicaciones/VII_ENCUESTA_NACIONAL_DROGAS_POBLACI oN_GENERAL_2019.pdf [Consulta: 26 oct 2020].
10. Teixeira V, Imbriago Y, Sarries E. Utilización de antidepresivos en pacientes ambulatorios del Hospital Policial. Rev Psiquiatr Urug 2015; 79 (1):39-48.

11. Uruguay. Ministerio de Salud Pública. Dispensación de medicamentos controlados en Uruguay. Periodo: enero-octubre 2018. MSP, 2018. Disponible en https://www.gub.uy/ministerio-salud-publica/sites/ministerio-salud-publica/files/documentos/publicaciones/Dispensaci $\% \mathrm{C} 3 \% \mathrm{~B} 3 \mathrm{n} \% 20$ medicamentos $\% 20$ controlados.pdf [Consulta: 26 oct 2020].

12. España. Ministerio de Sanidad, Servicios Sociales e Igualdad. Agencia Española de Medicamentos y Productos Sanitarios. Utilización de medicamentos antidepresivos en España durante el periodo 2000-2013. AEMPS, 2015. Disponible en: http://www.aemps.gob.es/medicamentosUsoHumano/observatorio/docs/antidepresivos-2000-2013.pdf [Consulta: 26 oct 2020].

13. Gusmão R, Quintão S. The use of antidepressants in Portugal: 1995-2009. Europ Psychiatr 2012; 27(Suppl 1):1.

14. Spence R, Roberts A, Ariti C, Bardsley M. Focus on: antidepressant prescribing. Trends in the prescribing of antidepressants in primary care. The Health Foundation, 2014. Disponible en: https://www.nuffieldtrust.org.uk/files/2018-10/qualitywatch-antidepressant-prescribing.pdf [Consulta: 26 oct 2020].

15. Pratt LA, Brody DJ, Gu Q. Antidepressant use in persons aged 12 and over: United States, 2005-2008. NCHS Data Brief 2011; (76):1-8.

16. Jirón M, Machado M, Ruiz I. Consumo de antidepresivos en Chile entre 1992 y 2004. Rev méd Chile 2008; 136(9):1147-54.

17. UdelaR. Facultad de Medicina. Departamento de Farmacología y Terapéutica. Observatorio de Medicamentos. Disponible en: http://www.farmacologia.hc.edu.uy/index.php?option $=$ com_content\&view $=$ article $\&$ id $=128$ [Consulta: 26 oct 2020].

18. Arnau J, Vallano A. Estudios de utilización de medicamentos. Medic Salud 2000; 3(2):72-7.

19. WHO Collaborating Centre for Drug Statistics Methodolog. ATC DDD Index. Disponible en: https://www.whocc.no/atc_ddd_index/?code=N06A [Consulta: 10 oct 2015].

20. Uruguay. Instituto Nacional de Estadística. Estimaciones y Proyecciones de Población. Disponible en: https:/www.ine.gub.uy/web/guest/estimaciones-y-proyecciones [Consulta: 10 mayo 2020].

21. Almada R, Miraballes R, Agrafojo S. Frecuencia de depresión en los pacientes que consultan en las policlínicas de medicina interna del Hospital de Clínicas Dr. Manuel Quintela. Rev Psiquiatr Urug 2014; 78(2):116-30.

22. Organización Panamericana de la Salud. "Depresión: hablemos", dice la OMS, mientras la depresión encabeza la lista de causas de enfermedad. Ginebra/Washington: OMS/OPS, 30 marzo 2017. Disponible en: https://www.paho.org/uru/index.php?option=com_content\&view $=$ article $\&$ id $=1152$ :de- 
presion-hablemos-dice-la-oms-mientras-la-depresion-encabeza-la-lista-de-causas-de-enfermedad\&Itemid $=451 \quad$ [Consulta: 27 oct 2020].

23. Uruguay. Ministerio de Salud Pública. Consumo de Benzodiazepinas y otros sicofármacos en territorio nacional. Periodo: Enero 2014 - Junio 2016. MSP, 2017. Disponible en: https://www.gub.uy/ministerio-salud-publica/sites/ministerio-salud-publica/files/documentos/publicaciones/Consumo\%20de $\% 20$ Benzodiacepinas $\% 20 \mathrm{y} \% 20$ otros $\% 20$ sicof $\%$ C3\%A1rmacos $\% 20$ en $\% 20$ territorio $\% 20$ nacional $\%$ C2\%A02017.pdf [Consulta: 26 oct 2020].

24. National Services Scotland. Medicines for mental health. Financial years 2003/04 to 2012/13. NHS, 24 september 2013. Disponible en: https://www.isdscotland.org/Health-Topics/Prescribing-and-Medicines/Publications/2013-09-24/2013-09-24-PrescribingMentalHealth-Report.pdf?40206545592 [Consulta: 22 oct 2020].

25. Trends in antidepressant consumption. (Presentado en el OECD Health Data National Correspondents meeting, Paris 23 October 2014). Disponible en: http://www.oecd.org/ els/health-systems/Item10_Trends-in-antidepressant-consumption_NIPH.pdf [Consulta: 27 oct 2020].

26. Brett J, Karanges EA, Daniels B, Buckley NA, Schneider C, Nassir A, et al. Psychotropic medication use in Australia, 2007 to 2015: changes in annual incidence, prevalence and treatment exposure. Aust N Z J Psychiatry 2017; 51(10): 990-9.

27. Pérez V, Speranza N, Tamosiunas G, Ormaechea G. Perfil de riesgo de los antidepresivos inhibidores selectivos de la recaptación de serotonina (ISRS). Rev Urug Med Int 2016; $1(3): 25-33$.

28. Ali MK, Lam RW. Comparative efficacy of escitalopram in the treatment of major depressive disorder. Neuropsychiatr Dis Treat 2011; 7:39-49.
29. Cipriani A, Santilli C, Furukawa TA, Signoretti A, Nakagawa A, McGuire H, et al. Escitalopram versus other antidepressive agents for depression. Cochrane Database Syst Rev 2009; (2):CD006532. doi: 10,1002/14651858.CD006532.pub2.

30. Simó Miñana J. [Use of prescription drugs in Spain and Europe]. Aten Primaria 2012; 44(6):335-47.

31. España. Ministerio de Sanidad, Agencia Española de Medicamentos y Productos Sanitarios. Agomelatina (Thymanax ${ }^{\circledR}$, Valdoxan ${ }^{\circledR)}$ y toxicidad hepática: nuevas recomendaciones de uso. AEMPS, 2014. Disponible en: https:// www.aemps.gob.es/informa/notasinformativas/medicamentosusohumano-3/seguridad-1/2014/ni-muh_fv_142014-agomelatina/ [Consulta: 27 oct 2020].

32. Pagano E. Perfil farmacológico de agomelatina, un nuevo antidepresivo. Bol Farmacol 2016; 7(2).

33. Kennedy SH, Lam RW, McIntyre RS, Tourjman SV, Bhat V, Blier P, et al. Canadian Network for Mood and Anxiety Treatments (CANMAT) 2016. Clinical guidelines for the management of adults with major depressive disorder: Section 3. Pharmacological treatments. Can J Psychiatry 2016; 61(9):540-60. Disponible en: https://www.cmh.org/sites/default/files/page-assets/programs-services/mentalhealth-services/mental-health-events-health-care-providers/canmat-pharmacological-39247.pdf [Consulta: 27 oct 2020].

34. Hansen RA, Gartlehner G, Lohr KN, Gaynes BN, Carey TS. Efficacy and safety of second-generation antidepressants in the treatment of major depressive disorder. Ann Intern Med 2005; 143(6):415-26

35. Pagano E, Domínguez V, Barboza L, Speranza N, Tamosiunas G. Mitos en el uso de antidepresivos(1) ¿Cuáles pacientes con depresión precisan un antidepresivo?. Bol Farmacol 2015; 6(3).

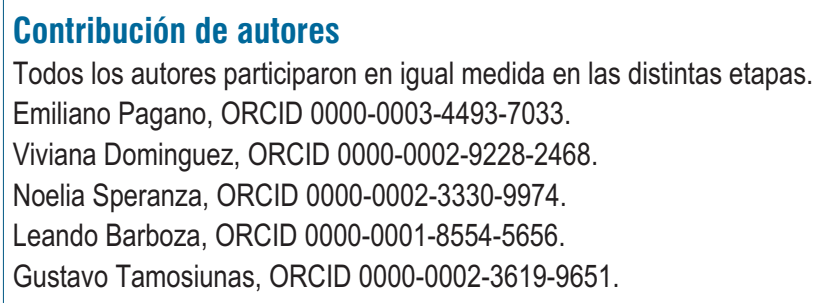

